\title{
ON EMBEDDING HIGHLY CONNECTED MANIFOLDS IN EUCLIDEAN SPACE
}

\author{
BY \\ J. B. MINKUS
}

\section{Chapter I. Introductory Remarks and Definitions}

1. Introduction. Let $M^{k}$ and $T^{k}$ be closed $k$-dimensional differentiable manifolds. A homeomorphism $h: M^{k} \rightarrow T^{k}$ will be called an almost diffeomorphism and we will say that $M^{k}$ and $T^{k}$ are almost diffeomorphic if $h \mid M^{k}$ $-\left\{x_{0}\right\}$ is a diffeomorphism for some point $x_{0}$ in $M^{k}$.

A closed $k$-dimensional differentiable manifold $M^{k}$ will be said to be almost differentiable embeddable in Euclidean $m$ space $R^{m}$ if $M^{k}$ is almost diffeomorphic to a manifold $T^{k}$ which is differentiably embeddable in $R^{m}$.

The following example is illustrative: Let $\theta^{k}$ denote the group of differentiable manifolds homeomorphic to the standard $k$-sphere $S^{k}$. It is well known that every manifold $M^{k} \in \theta^{k}(k \geqq 7)$ is almost diffeomorphic to $S^{k}$ (see Smale [13]) and therefore every such manifold is almost differentiably embeddable in $R^{k+1}$. It follows from [13, Theorem 1.1] that $M^{k} \in \theta^{k}$ is differentiably embeddable in $R^{k+1}$ if and only if $M^{k}$ is diffeomorphic to $S^{k}(k \geqq 7)$.

In this paper proofs of the following facts will be given:

Theorem 1. Let $n$ be an even integer, $n \geqq 6$. Let $M^{2 n}$ be a closed $(n-1)$ connected $2 n$-dimensional differentiable manifold. Then $M^{2 n}$ is almost differentiably embeddable in $R^{2 n+1}$ if and only if the index $I\left(M^{2 n}\right)$ of $M^{2 n}$ is zero and $M^{2 n}$ is $n$-parallelizable.

A differentiable manifold is called a $\pi$-manifold if the Whitney sum of its tangent bundle with a trivial line bundle is trivial.

Corollary 1. A closed $(n-1)$-connected $2 n$-dimensional differentiable manifold ( $n$ even, $n \geqq 6$ ) is almost differentiably embeddable in $R^{2 n+1}$ if and only if it is a $\pi$-manifold.

Theorem 2. Let $n \equiv 3,5,7(\bmod 8), n \geqq 5$. Let $M^{2 n}$ be a closed $(n-1)$ connected 2 -dimensional differentiable manifold. Then $M^{2 n}$ is almost differentiably embeddable in $R^{2 n+1}$ if and only if the Arf invariant $\Phi\left(M^{2 n}\right)$ of $M^{2 n}$ (defined below) is zero.

It has been conjectured by various researchers that if $n$ is odd the Arf invariant of a closed $(n-1)$-connected $2 n$-dimensional differentiable manifold is always zero. Some additional results without any restrictions on

Received by the editors September 20, 1963. 
the Arf invariant are also obtained in this paper (see Theorem 3 below) $\left({ }^{1}\right)$.

2. The picture to keep in mind. The proofs of Theorems 1 and 2 are generalizations of the following "proof" of the fact that the 2-dimensional torus $T^{2}$ is embeddable in 3-space. If we take out the interior of a small disk $D_{0}^{2} \subset T^{2}$ then what is left, $N^{2}=T^{2}-\operatorname{int} D_{0}^{2}$, is a handlebody. More precisely $N^{2}$ is a disk $D^{2}$ with two handles $D_{1}^{1} \times D^{1}, D_{2}^{1} \times D^{1}$ attached along the tubular neighborhoods of two linked zero spheres, $S_{1}^{0}, S_{2}^{0} \subset \partial D^{2}$ (see Milnor [8, Introduction]).

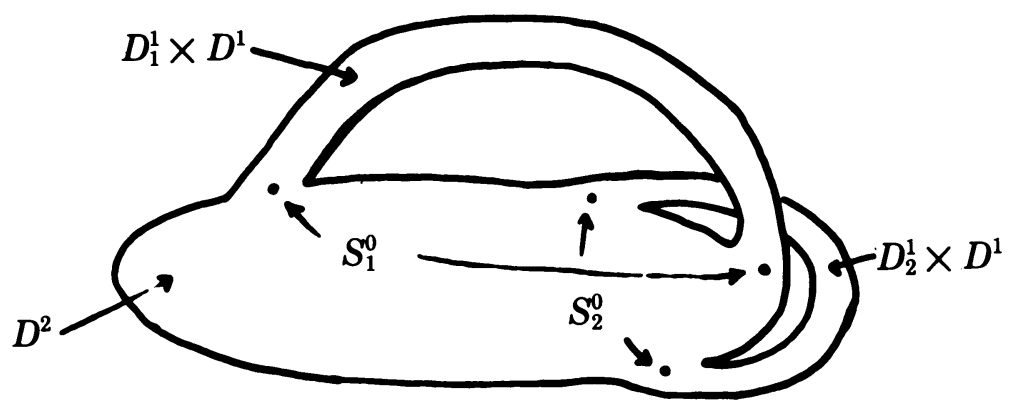

Figure 1

The handles are attached without twisting (that is, there is no Möbius strip embedded in Figure 1).

Let Euclidean 3-space $R^{3}$ be decomposed into the direct sum of two subspaces $R^{3}=R^{2} \oplus R^{1}$. Let

$$
\begin{aligned}
& R_{+}^{3}=\left\{R^{2} \oplus x \mid x>0\right\}, \\
& R_{-}^{3}=\left\{R^{2} \oplus x \mid x<0\right\} .
\end{aligned}
$$

Define an embedding $f$ of $N^{2}=D^{2} \cup D_{1}^{1} \times D^{1} \cup D_{2}^{1} \times D^{1}$ into $R^{3}$ as follows:

Look at $D^{2}$ as the set of all pairs of real numbers $(x, y)$ such that $x^{2}$ $+y^{2} \leqq 1$.

Let $f \mid D^{2}$ be defined by

$$
f(x, y)=\left(x, y, x^{2}+y^{2}-1\right) .
$$

Then $f$ maps $\partial D^{2}$ into the unit circle in $R^{2}$ and it maps int $D^{2}$ into $R_{-}^{3}$. Now embed the handles $D_{1}^{1} \times D^{1}$ and $D_{2}^{1} \times D^{1}$ in $R^{2}$ by embedding

(1) Added in proof. This research was submitted to the Division of Physical Sciences of the University of Chicago in partial fulfillment of the requirements for the degree of Doctor of Philosophy. Both the author and R. V. DeSapio have extended these results to $\pi$-manifolds of lower connectivity (see DeSapio, Embedding $\pi$-manifolds, Thesis, Univ. of Chicago, Chicago, Ill., 1964). 
(int $\left.D_{1}^{1}\right) \times D^{1}$ in the unbounded component of $R^{2}-f\left(\partial D^{2}\right)$ and embedding (int $\left.D_{2}^{1}\right) \times D^{1}$ in the bounded component. We have to do this to make sure that the handles do not intersect in $R^{2}$ since $S_{1}^{0}$ and $S_{2}^{0}$ are linked in $\partial D^{2}$. Note also that the fact that the handles are attached without twisting is important. We could not have embedded them in $R^{2}$ (compatibly with $\left.f \mid D^{2}\right)$ if they had been attached with a twist.

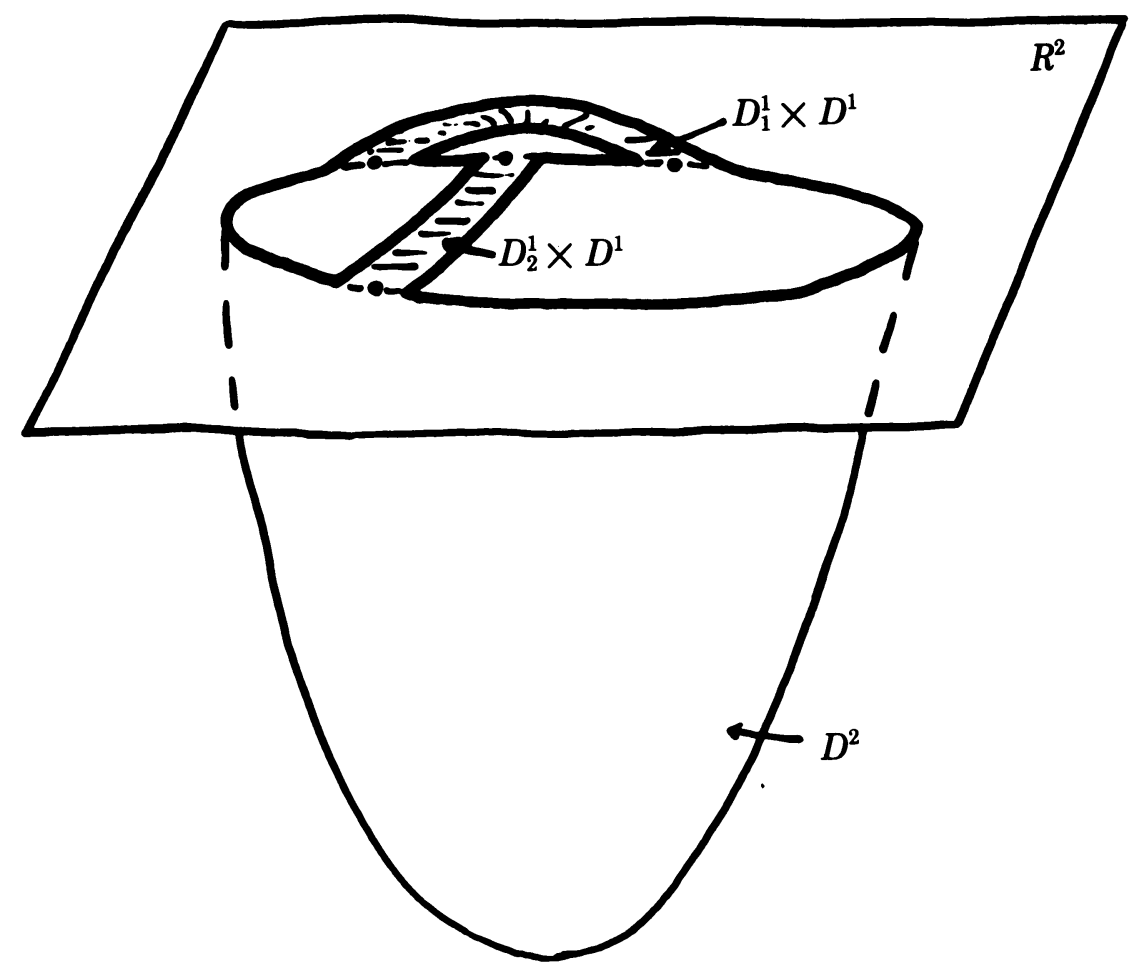

FIGURE 2

Thus $f: N^{2} \rightarrow R^{3}$ is an embedding such that $f\left(N^{2}\right) \cap R^{2}=f\left(N^{2}-\operatorname{int} D^{2}\right)$ and $f$ (int $\left.D^{2}\right) \subset R_{-}^{3}$ (see Figure 2). Note that $\partial N^{2}$ is a circle (as it must be since we started by taking out the interior of a disk $D^{2} \subset T^{2}$ ) and that $f$ embeds $\partial N^{2}$ in $R^{2}$ ("thickened" curve in Figure 2). Now $f\left(\partial N^{2}\right)$ is not the unit circle in $R^{2}$ but it bounds something homeomorphic to a disk $D_{0}^{2} \subset R^{2}$. Lift int $D_{0}^{2}$ off $R^{2}$ into $R_{+}^{3}$ to get an embedding $T^{2} \subset R^{3}$.

The above is the geometrical motivation for what follows. The proofs of Theorems 1 and 2 rely heavily on the fact that an $(n-1)$-connected $2 n$-dimensional differentiable manifold $M^{2 n}$ becomes a handlebody if we remove the interior of a small disk from the manifold (Smale [12]). It will be shown that the hypotheses of these theorems are good enough to ensure that this handlebody can be embedded in $R^{2 n+1}$ in an analogous 
fashion to $f: N^{2} \subset R^{3}$. Smale's version of the Schoenflies theorem will then be used to "put the cap back" on this handlebody in $R^{2 n+1}$ giving an almost differentiable embedding $M^{2 n} \subset R^{2 n+1}$.

3. Definitions and notation. Much of the material in this section will be found in Smale [12], [14] and Wall [17].

Let $M^{2 n}$ be a closed $(n-1)$-connected $2 n$-dimensional differentiable manifold, $n \geqq 3$.

Let $D_{0}^{2 n}$ be a differentiably embedded disk $D_{0}^{2 n} \subset M^{2 n}$. Smale has shown (see [12, Theorem G]) that $M^{2 n}-\operatorname{int} D_{0}^{2 n}$ is a handlebody in $\mathscr{Z}(2 n, k, n)$ where $k=\operatorname{rank} H_{n}\left(M^{2 n}, Z\right)$ and $\mathscr{Z}(2 n, k, n)$ is defined as follows:

Let $D_{k}=\bigcup_{i=1}^{k} D_{i}^{n} \times D^{n}=$ disjoint union of $k$ copies of $D^{n} \times D^{n}$, where $D^{m}$ (or $D_{i}^{m}$ ) denotes the closed disk of dimension $m$.

Let $C_{k}=\bigcup_{i=1}^{k} \partial D_{i}^{n} \times D^{n}\left(C_{k} \subset D_{k}\right)$.

Let $\lambda: C_{k} \rightarrow \partial D^{2 n}$ be a differentiable embedding.

Let $N^{2 n}=D^{2 n} \cup_{\lambda} D_{k}$. There is a unique differentiable structure on $N^{2 n}$ compatible with the structures on $D^{2 n}$ and $D^{k}$. In Smale's terminology the differentiable manifold $N^{2 n}$ is a handlebody with presentation $\lambda$ and the elements of $\mathscr{Z}(2 n, k, n)$ consist of the diffeomorphism classes of all manifolds obtained in this way as $\lambda$ varies. Note that a given handlebody could conceivably have two different (that is, not diffeotopic) presentations.

Given a presentation $\lambda$ of a handlebody $N^{2 n}$ in $\mathscr{U}(2 n, k, n)$ we now define three new quantities: $L(\lambda)$, the linking matrix of $\lambda ; B(\lambda)$, the canonical basis of $H_{n}\left(N^{2 n}, Z\right)$ associated with $\lambda ; \alpha(\lambda)$, the "twisting coefficients" of $\lambda . L(\lambda)=\left\|c_{i j}\right\|$ is the $k \times k$ integer-valued matrix defined by $c_{i i}=0$, $i=1, \cdots, k$, and

$$
c_{i j}=\operatorname{Lk}\left(\lambda\left(\partial D_{i}^{n} \times 0\right), \lambda\left(\partial D_{j}^{n} \times 0\right)\right), \quad i \neq j,
$$

where $\operatorname{Lk}(\alpha, \beta)$ is the linking number of two $(n-1)$-dimensional cycles $\alpha, \beta$ in an oriented $(2 n-1)$-sphere. $L(\lambda)$ is a symmetric matrix $\left(c_{i j}=c_{j i}\right)$ if $n$ is even and a skew symmetric matrix $\left(c_{i j}=-c_{j i}\right)$ if $n$ is odd. (See Siefert and Threlfall [11, pp. 277-282] for definitions and properties of $\operatorname{Lk}(\alpha, \beta)$.)

$B(\lambda)$ is defined as follows: For each $i=1, \cdots, k$ there is an embedding $g_{i}: D_{i}^{n} \rightarrow \partial D^{2 n}$ such that $g_{i}\left|\partial D_{i}^{n}=\lambda\right| \partial D_{i}^{n} \times 0$ (see Lemma 2 below). The subset $S_{i}^{n}=g_{i}\left(D_{i}^{n}\right) \cup D_{i}^{n} \times 0 \subset N^{2 n}$ is an embedded $n$-sphere in $N^{2 n}$. The homology classes carried by $\left\{S_{i}^{n}\right\}$ define a free basis for $H_{n}\left(N^{2 n}, Z\right)$ depending only on $\lambda$ which we denote by $B(\lambda)$.

Note that the intersection number of the homology classes carried by $S_{i}^{n}$ and $S_{j}^{n}(i \neq j)$ is obtained by looking at the geometrical intersection

$$
S_{i}^{n} \cap S_{j}^{n}=g_{i}\left(D_{i}^{n}\right) \cap g_{j}\left(D_{j}^{n}\right) .
$$

(See Seifert and Threlfall [11, pp. 246-248] for definitions and properties 
of intersection numbers.) Since the linking number of $\lambda\left(\partial D_{i}^{n} \times 0\right)$ and $\lambda\left(\partial D_{j}^{n} \times 0\right)$ is also obtained by looking at $g_{i}\left(D_{i}^{n}\right) \cap g_{j}\left(D_{j}^{n}\right)$ we have that the intersection number of $\left[S_{i}^{n}\right]$ and $\left[S_{j}^{n}\right]$ in $H_{n}\left(N^{2 n}, Z\right)$ is equal to

$$
\operatorname{Lk}\left(\lambda\left(\partial D_{i}^{n} \times 0\right), \lambda\left(\partial D_{j}^{n} \times 0\right)\right), \quad i \neq j .
$$

This then gives another way of determining $L(\lambda)$ which will be useful later.

It remains to define $\alpha(\lambda)$. This is done as follows: Let $\bar{h}$ denote the inclusion $\bar{h}_{i}: S_{i}^{n} \subset N^{2 n}$. Since $N^{2 n}$ is $(n-1)$-connected, $n \geqq 3$, we have by results of Haefliger (see [ 3 , Theorems 4.1 and 5.1]) that $\bar{h}_{i}$ is homotopic to a differentiable embedding $h_{i}: S^{n} \rightarrow N^{2 n}$ and further any two such differentiable embeddings $h_{i}$ and $h_{i}^{\prime}$ are diffeotopic. That is, $h_{i}\left(S^{n}\right)$ and $h_{i}^{\prime}\left(S^{n}\right)$ have equivalent normal bundles in $N^{2 n}$. Thus $\lambda \mid \partial D_{i}^{n} \times D^{n}$ determines a unique element of $\pi_{n-1}(\mathrm{SO}(n))$ the elements of which are in one-to-one correspondence with $n$-dimensional vector bundles over $S^{n}$ (see Steenrod [15, Theorem 18.6]). Let $\alpha\left(\lambda_{i}\right) \in \pi_{n-1}(\operatorname{SO}(n))$ denote this element. Then

$$
\alpha(\lambda)=\left(\alpha\left(\lambda_{1}\right), \alpha\left(\lambda_{2}\right), \cdots, \alpha\left(\lambda_{k}\right)\right) \in \sum_{i=1}^{k} \pi_{n-1}(\operatorname{SO}(n)) .
$$

$\alpha(\lambda)$ is just a measure of how twisted the handles $D_{k}$ are in $D^{2 n} \cup_{\lambda} D_{k}$.

The following proposition will be needed in Chapter II. It is an easy consequence of Smale [12, Theorem M] and Smale [13, Theorem 1.4] (or Munkres [10, Theorem 6.10]).

Proposition. Let $k>7$. Two closed $k$-dimensional differentiable manifolds $M^{k}$ and $T^{k}$ are almost diffeomorphic if and only if there exists $H^{k} \in \theta^{k}$ such that $T^{k}$ is the connected sum of $M^{k}$ and $H^{k}\left(T^{k}=M^{k} \# H^{k}\right)$. (See Milnor [7] or Kervaire and Milnor [5] for the definition of connected sum.)

Another piece of notation which will be used is the following: Let $U$ be a square matrix. The $\operatorname{symbol} \operatorname{diag}(U, U, \ldots, U)$ will denote the matrix

$$
\left(\begin{array}{cccccc}
U & & & & \\
& U & & & 0 & \\
& & \cdot & & & \\
& 0 & & & \cdot & U
\end{array}\right) .
$$

\section{Chapter II. Proofs of Theorems 1 and 2}

1. The main lemma. Let Euclidean $(2 n+1)$-space be decomposed into the direct sum of a line and a hyperplane $R^{2 n+1}=R^{2 n} \oplus R^{1}$. Let

$$
\begin{aligned}
& R_{+}^{2 n+1}=\left\{R^{2 n} \oplus x \mid x>0\right\}, \\
& R_{-}^{2 n+1}=\left\{R^{2 n} \oplus x \mid x \leqq 0\right\} .
\end{aligned}
$$

The proofs of Theorems 1 and 2 depend upon the following lemma. 
LEMma 1. Let $N^{2 n}$ be an element of $\mathscr{L}(2 n, k, n), n \geqq 5$. Suppose $N^{2 n}$ has a presentation $\lambda$ such that $\alpha(\lambda)=0$ and $L(\lambda)=\operatorname{diag}(U, U, \ldots, U)$ where $U$ is a $2 \times 2$ integer-valued matrix. Then there is a differentiable embedding $f: N^{2 n} \rightarrow R_{-}^{2 n+1}$ such that $f\left(\partial N^{2 n}\right) \subset R^{2 n}$.

The proof of Lemma 1 depends in turn upon the following.

Lemma 2. Given a differentiable embedding $\lambda: C_{k} \rightarrow \partial D^{2 n}, n \geqq 5, k=2 s$, if $L(\lambda)=\operatorname{diag}(U, U, \ldots, U)$ for some $2 \times 2$ integer-valued matrix $U$ then there exist differentiable embeddings $g_{i}: D_{i}^{n} \rightarrow \partial D^{2 n}, i=1, \cdots, k$, such that

(i) $g_{i}\left|\partial D_{i}^{n}=\lambda\right| \partial D_{i}^{n} \times 0$,

(ii) $g_{i}\left(D_{i}^{n}\right) \cap g_{j}\left(D_{j}^{n}\right)=\emptyset$ unless $i=2 l-1, j=2 l$ for some $1 \leqq l \leqq s$. (In other words, these embedded disks intersect in pairs.)

Note that Lemma 2 is false in the case $n=2$. There are three circles disjointly embedded in the three spheres $S_{1}^{1}, S_{2}^{1}, S_{3}^{1} \subset S^{3}$ such that $\operatorname{Lk}\left(S_{i}^{1}, S_{j}^{1}\right)$ $=0, i \neq j$, but the inclusion map $f: S_{1}^{1} \rightarrow S^{3}-\left(S_{2}^{1} \cup S_{3}^{1}\right)$ gives a nonzero element of $\pi_{1}\left(S^{3}-\left(S_{2}^{1} \cup S_{3}^{1}\right)\right.$ ) (see Figure 3 ).

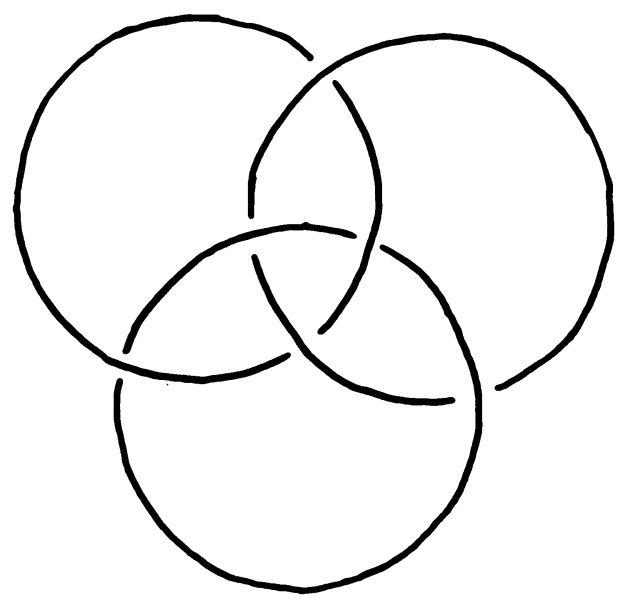

Figure 3

Proof of Lemma 2. Let $X_{l}=\partial D^{2 n}-\bigcup_{i \neq 2-1,2 l} \lambda\left(\partial D_{i}^{n} \times 0\right), l=1 \cdots, s$. Then

$$
\lambda\left|\partial D_{2 l-1}^{2 n} \times 0: \partial D_{2 l-1}^{2 n} \times 0 \rightarrow X_{l}, \quad \lambda\right| \partial D_{2 l}^{2 n} \times 0: \partial D_{2 l}^{2 n} \times 0 \rightarrow X_{l}
$$

Since $L(\lambda)=\operatorname{diag}(U, U, \cdots, U)$ for some $2 \times 2$ matrix $U$ we know that $\lambda\left(\partial D_{2 l-1}^{n} \times 0\right)$ and $\lambda\left(\partial D_{2 l}^{n} \times 0\right)$ are linked with none of the other $\lambda\left(\partial D_{i}^{n} \times 0\right)$. It will now. be shown that this implies that the two maps written above are nullhomotopic in $X_{l}$. To see this observe that

$$
\begin{aligned}
\operatorname{Lk}\left(\lambda\left(\partial D_{2 l-1}^{n} \times 0\right),\right. & \left.\bigcup_{i \neq 2 L-1,2 l} \lambda\left(\partial D_{i}^{n} \times 0\right)\right) \\
& =\sum_{i \neq 2-1,2 l} \operatorname{Lk}\left(\lambda\left(\partial D_{2 l-1}^{n} \times 0\right), \lambda\left(\partial D_{i}^{n} \times 0\right)\right)=0
\end{aligned}
$$


(see $[11$, p. 278]). Similarly,

$$
\operatorname{Lk}\left(\lambda\left(\partial D_{2 l}^{n} \times 0\right), \bigcup_{i \neq 2 l-1,2 l} \lambda\left(\partial D_{i}^{n} \times 0\right)\right)=0 .
$$

Thus by the definition of linking number $\lambda\left(\partial D_{2 l-1}^{n} \times 0\right)$ and $\lambda\left(\partial D_{2 l}^{n} \times 0\right)$ both bound chains in the complement of $\bigcup_{i \neq 2 l-1,2 l} \lambda\left(\partial D_{i}^{n} \times 0\right)$. In other words, they both bound chains in $X_{l}$.

Further, $X_{l}$ is simply connected if $n \geqq 3$. To see this let $S^{1} \subset X_{l}$ be a differentiably embedded circle. This circle is nullhomotopic in $\partial D^{2 n}$ and so $S^{1} \subset \partial D^{2 n}$ bounds a differentiably embedded disk $D^{2} \subset \partial D^{2 n}$. Now $(n-1)$ $+2<2 n-1$ since $n \geqq 3$. Therefore since $\bigcup_{i \neq 2 l-1,2} \lambda\left(\partial D_{i}^{n} \times 0\right)$ is $(n-1)$ dimensional we can assume this disk does not intersect $\bigcup_{i \neq 2-1,2 l} \lambda\left(\partial D_{i}^{n} \times 0\right)$ as desired.

Now, by the Alexander Duality Theorem,

$$
H^{p}\left(\bigcup_{i \neq 2 l-1,2 l} \lambda\left(\partial D_{i}^{n} \times 0\right), Z\right) \approx \widetilde{H}_{2 n-p-2}\left(X_{l}, Z\right) .
$$

Therefore $H_{i}\left(X_{l}, Z\right)=0, i \leqq n-2$. Therefore, by the Hurewicz Isomorphism Theorem, $\pi_{n-1}\left(X_{l}\right) \approx H_{n-1}\left(X_{l}, Z\right)$.

But we have shown that $\lambda \mid \partial D_{2 l-1}^{n} \times 0$ and $\lambda \mid \partial D_{2 l}^{n} \times 0$ are both nullhomologous in $X_{l}$. So they are both nullhomotopic in $X_{l}, l=1, \cdots, s$.

Thus we have proven the existence of a continuous map $\bar{g}_{1}: D_{1}^{n} \rightarrow X_{1}$ such that $\bar{g}_{1}\left|\partial D_{1}^{n}=\lambda\right| \partial D_{1}^{n} \times 0$. To find a differentiable embedding $g_{1}$ with the same property proceed as follows: Since $n \geqq 5$ we can, using Haefliger [3], approximate $\bar{g}_{1}$ by a differentiable embedding $h: D_{1}^{n} \rightarrow X_{1}$. Then using results of Thom and Haefliger (see Introduction to [3]) we construct a diffeomorphism $\phi: X_{1} \rightarrow X_{1}$ such that $\bar{g}_{1} \mid \partial D_{1}^{n}=\phi \circ\left(h \mid \partial D_{1}^{n}\right)$. The differentiable embedding $g_{1}: D_{1}^{n} \rightarrow X_{1}$ is now defined by $g_{1}(x)=\phi \circ h(x)$ for all $x \in D_{1}^{n}$. Clearly $g_{1}\left|\partial D_{1}^{n}=\lambda\right| \partial D_{1}^{n} \times 0$.

In the same way from the existence of a continuous map $\bar{g}_{2}: D_{2}^{n} \rightarrow X_{1}$ satisfying $\bar{g}_{2}\left|\partial D_{2}^{n}=\lambda\right| \partial D_{2}^{n} \times 0$ the existence of a differentiable embedding $g_{2}: D_{2}^{n} \rightarrow X_{1}$ with the same property can be proven.

A differentiable embedding $g_{3}: D_{3}^{n} \rightarrow X_{2}$ such that $g_{3}\left|\partial D_{3}^{n}=\lambda\right| \partial D_{3}^{n} \times 0$ can be defined as above. Now, however, we must make sure that $g_{3}\left(D_{3}^{n}\right)$ does not intersect $g_{1}\left(D_{1}^{n}\right) \cup g_{2}\left(D_{2}^{n}\right)$ so that condition (ii) of the lemma will be satisfied. This is done as follows: We know that $\lambda \mid \partial D_{3}^{n} \times 0$ : $\partial D_{3}^{n} \times 0 \rightarrow X_{2}$ is nullhomotopic. Claim further that $\lambda \mid \partial D_{3}^{n} \times 0: \partial D \rightarrow X_{2}$ $-\left(g_{1}\left(D_{1}^{n}\right) \cup g_{2}\left(D_{2}^{n}\right)\right)$ is nullhomotopic. To see this pick a point $p_{2} \in g_{1}\left(D_{1}^{n}\right)$ $\cap g_{2}\left(D_{2}^{n}\right)$ (which must be nonempty if $\operatorname{Lk}\left(\lambda\left(\partial D_{1}^{n} \times 0\right), \lambda\left(\partial D_{2}^{n} \times 0\right)\right) \neq 0$ as it will be in the application of this lemma; for completeness, however, if $g_{1}\left(D_{1}^{n}\right) \cap g_{2}\left(D_{2}^{n}\right)=\emptyset$ pick two points $\left.p_{2} \in g_{1}\left(D_{1}^{n}\right), p_{2}^{\prime} \in g_{2}\left(D_{2}^{n}\right)\right)$. Then $X_{2}-\left(g_{1}\left(D_{1}^{n}\right) \cup g_{2}\left(D_{2}^{n}\right)\right)$ is a deformation retract of $X_{2}-\left\{p_{2}\right\}$ (or $X_{2}$ $\left.-\left\{p_{2}, p_{2}^{\prime}\right\}\right)$. But $\lambda \mid \partial D_{3}^{n} \times 0: 2 D_{3}^{n} \times 0 \rightarrow X_{2}-\left\{p_{2}\right\}$ is nullhomotopic and thus $\lambda \mid \partial D_{3}^{n} \times 0: \partial D_{3}^{n} \times 0 \rightarrow X_{2}-\left(g_{1}\left(D_{1}^{n}\right) \cup g_{2}\left(D_{2}^{n}\right)\right)$ is nullhomotopic. 
Therefore, as before, we can find a differentiable embedding

$$
g_{3}: D_{3}^{n} \rightarrow X_{2}-\left(g_{1}\left(D_{1}^{n}\right) \cup g_{2}\left(D_{2}^{n}\right)\right) \quad \text { with } g_{3}\left|\partial D_{3}^{n}=\lambda\right| \partial D_{3}^{n} \times 0 \text {. }
$$

Continue in this way to define $g_{4}, \cdots, g_{k}$ and Lemma 2 is proved.

Proof of Lemma 1. Recall that $N^{2 n}=D^{2 n} \cup_{\lambda} D_{k}$. A topological embedding $f^{\prime}: N^{2 n} \rightarrow R_{-}^{2 n+1}$ having the desired properties will now be constructed. Look at $D^{2 n}$ as $2 n$-tuples of real numbers $\left(x_{1}, \cdots, x_{2 n}\right)$ such that $\sum_{i=1}^{2 n} x_{i}^{2}$ $\leqq 1$.

Define $f^{\prime} \mid D^{2 n}: D^{2 n} \rightarrow R_{-}^{2 n+1}$ by the formula

$$
f^{\prime}\left(x_{1}, \cdots, x_{2 n}\right)=\left(x_{1}, \cdots, x_{2 n}, \sum_{i=1}^{2 n}\left(x_{i}^{2}-1\right)\right) .
$$

Note that $f\left(\partial D^{2 n}\right)$ is the unit $(2 n-1)$-sphere $S^{2 n-1} \subset R^{2 n}$.

Extend $f^{\prime}$ to $D^{2 n} \cup_{\lambda} \bigcup_{i=1}^{k} D_{i}^{n} \times 0$ as follows: Since by hypothesis $L(\lambda)$ $=\operatorname{diag}(U, U, \ldots, U)$ we can by Lemma 2 find differentiable embeddings $g_{i}: D_{i}^{n} \rightarrow \partial D^{2 n}$ such that (i) $g_{i}\left|\partial D_{i}^{n}=\lambda\right| \partial D_{i}^{n} \times 0$ and (ii) the $\left\{g_{i}\left(D_{i}^{n}\right)\right\}$ intersect in pairs. The points $y \in f^{\prime}\left(g_{i}\left(D_{i}^{n}\right)\right) \subset S^{2 n-1} \subset R^{2 n}, i=1, \cdots, k$, can be looked at as unit vectors in $R^{2 n}$ and so it makes sense to multiply them by scalars. Let $\gamma:[0,1] \rightarrow R$ be a real-valued function satisfying

(i) $\gamma$ is $C^{\infty}$ on $[0,1)$,

(ii) $d \gamma(0) / d t=0, d \gamma(1) / d t=-\infty$,

(iii) $\gamma(0)=1 / 2, \gamma(1)=0$,

(iv) $\gamma$ is decreasing on $[0,1]$.

Let $x$ be a point in $D_{i}^{n} \times 0 \subset N^{2 n}$ and let $\|x\|$ be the norm of $x$ looked at as a vector in the unit ball $D_{i}^{n} \times 0$. Define $f^{\prime} \mid D_{i}^{n} \times 0: D_{i}^{n} \times 0 \rightarrow R^{2 n}$ by the formula

$$
f^{\prime}(x)= \begin{cases}(1+\gamma(\|x\|)) f^{\prime}\left(g_{i}(x)\right), & i \text { odd, } \\ (1-\gamma(\|x\|)) f^{\prime}\left(g_{i}(x)\right), & i \text { even. }\end{cases}
$$

(This is just a way of saying embed half of the $\left\{D_{i}^{n} \times 0\right\}$ in the bounded component of $R^{2 n}-S^{2 n-1}$ and half in the unbounded component.)

Since $g_{i}\left|\partial D_{i}^{n}=\lambda\right| \partial D_{i}^{n} \times 0$ we know that $f^{\prime}$ is defined on $D^{2 n} \cup_{\lambda} \bigcup_{i=1}^{k} D_{i}^{n}$ $\times 0$. Since the $\left\{g_{i}\left(D_{i}^{n}\right)\right\}$ intersect in pairs we know that

$$
f^{\prime}: D^{2 n} \cup_{\lambda} \bigcup_{i=1}^{k} D_{i}^{n} \times 0 \rightarrow R_{-}^{2 n+1}
$$

is an embedding. Further

$$
f\left(\partial D^{2 n} \cup_{\lambda} \bigcup_{i=1}^{k} D_{i}^{n} \times 0\right) \subset R^{2 n} .
$$

It remains to extend $f^{\prime}$ to the rest of $D_{k}$ (in other words, we want to "thicken the cores" $D_{i}^{n} \times 0$ of the handles $D_{i}^{n} \times D^{n}$ in $\left.R^{2 n}\right)$. This is where 
the condition $\alpha(\lambda)=0$ comes in.

Let $E\left(\nu_{i}^{n}\right) \subset R^{2 n}$ be the total space of the normal tube $\nu_{i}^{n}$ of $f^{\prime}\left(D_{i}^{n} \times 0\right)$ in $R^{2 n}$ whose radius is chosen so that $E\left(\nu_{i}^{n}\right) \cap f^{\prime}\left(\partial D^{2 n}\right)=f^{\prime}\left(\lambda\left(\partial D_{i}^{n} \times D^{n}\right)\right)$. (We can assume without loss of generality that the radius of $D^{n}$ is small enough so that $E\left(\nu_{i}^{n}\right) \cap E\left(\nu_{j}^{n}\right)=\emptyset(i \neq j)$.)

Let $p_{i}: D_{i}^{n} \times D^{n} \rightarrow E\left(\nu_{i}^{n}\right)$ be a diffeomorphism defining a product structure on $\nu_{i}^{n}$ (in particular, $p_{i}\left|D_{i}^{n} \times 0=f^{\prime}\right| D_{i}^{n} \times 0$ ). Look at $\bar{p}_{i}=p_{i} \mid \partial D_{i}^{n}$ $\times D^{n}: \partial D_{i}^{n} \times D^{n} \rightarrow E\left(\nu_{i}^{n} \mid \partial D_{i}^{n}\right)$. Note that $E\left(\nu_{i}^{n} \mid \partial D_{i}^{n}\right)=f^{\prime}\left(\lambda\left(\partial D_{i}^{n} \times D^{n}\right)\right)$. Therefore $\left(f^{\prime}\right)^{-1} \circ \bar{p}_{i}$ and $\lambda \mid \partial D_{i}^{n} \times D^{n}$ both define product structures on the tubular neighborhood $\lambda\left(\partial D_{i}^{n} \times D^{n}\right)$ of $\lambda\left(\partial D_{i}^{n} \times 0\right) \subset \partial D^{2 n}$. Now by the "tubular neighborhood theorem" (see Wall [17, bottom of p. 164]) there is a diffeotopy

$$
F:\left(\partial D_{i}^{n} \times D^{n}\right) \times I \rightarrow \lambda\left(\partial D_{i}^{n} \times D^{n}\right)
$$

between $\left(f^{\prime}\right)^{-1} \circ \bar{p}_{i}$ and some product structure

$$
t_{i}: \partial D_{i}^{n} \times D^{n} \rightarrow \lambda\left(\partial D_{i}^{n} \times D^{n}\right),
$$

where $t_{i}$ is related to $\lambda \mid \partial D_{i}^{n} \times D^{n}$ by a map $s_{i}: \partial D_{i}^{n} \rightarrow \operatorname{SO}(n)$ such that

$$
t_{i}(x, y)=\lambda\left(x, s_{i}(x) \circ y\right) .
$$

(Essentially this says that the product structure $\left(f^{\prime}\right)^{-1} \circ \bar{p}_{i}$ differs from $\lambda \mid \partial D_{i}^{n} \times D^{n}$ by the action of $\mathrm{SO}(n)$.)

It is easily seen that the homotopy class $\left[s_{i}\right] \in \pi_{n-1}(\mathrm{SO}(n))$ is precisely the characteristic class $\alpha\left(\lambda_{i}\right)$ of the normal bundle of the embedded sphere $h\left(S_{i}^{n}\right) \subset N^{2 n}$ defined in Chapter $\mathrm{I}$ and so

$$
\alpha(\lambda)=\left(\left[s_{1}\right],\left[s_{2}\right], \cdots,\left\lfloor s_{k}\right\rfloor\right) \in \pi_{n-1}(\mathrm{SO}(n)) .
$$

Now by hypothesis, $\alpha(\lambda)=0$. Therefore each $s_{i}$ is homotopic to the constant map. This homotopy supplies us with a diffeotopy between $t_{i}$ and $\lambda \mid \partial D_{i}^{n} \times D^{n}$ and so with a diffeotopy betweem $\left(f^{\prime}\right)^{-1} \circ \bar{p}_{i}$ and $\lambda \mid \partial D_{i}^{n}$ $\times D^{n}$. Let $\bar{p}$ be the union of the maps $\bar{p}_{i}$. Then $\left(f^{\prime}\right)^{-1} \circ \bar{p}$ is diffeotopic to $\lambda$ and therefore the handlebody $D^{2 n} \cup_{\left(f^{\prime}\right)-1_{0} \bar{p}} D_{k}$ is diffeomorphic to $N^{2 n}$ $=D^{2 n} \cup_{\lambda} D_{k}$. Further since

$$
\left(f^{\prime}\right)^{-1} \circ \bar{p}\left|\partial D_{i}^{n} \times 0=\lambda\right| \partial D_{i}^{n} \times 0, \quad i=1, \cdots, k,
$$

the map

$$
f^{\prime}: D^{2 n} \cup_{\left(f^{\prime}\right)-1_{\circ} \bar{p}} \bigcup_{i=1}^{k} D_{i}^{n} \times 0 \rightarrow R_{-}^{2 n+1}
$$

is defined. Now $f^{\prime}$ can be extended to the rest of $D_{k}$ by $f^{\prime} \mid D_{i}^{n} \times D^{n}=p_{i}$. Thus we have constructed a topological embedding $f^{\prime}: N^{2 n} \rightarrow R_{-}^{2 n+1}$ such that $f^{\prime}\left(\partial N^{2 n}\right) \subset R^{2 n}$. Smooth $f^{\prime}$ at the "corners" to obtain a differentiable embedding $f$ proving Lemma 1 . 
2. Auxiliary lemmas. In this section we investigate the conditions under which the hypotheses of Lemma 1 will be satisfied.

Lemma 3. Let $M^{2 n}$ be a closed $(n-1)$-connected $2 n$-dimensional differentiable manifold ( $n$ even, $n \neq 2$ ) and suppose the index $I\left(M^{2 n}\right)=0$ and $M^{2 n}$ is n-parallelizable, Let $D_{0}^{2 n} \subset M^{2 n}$ be a differentiably embedded disk and let $N^{2 n}=M^{2 n}-\operatorname{int} D_{0}^{2 n}$. Then $N^{2 n}$ has a presentation $\lambda$ such that

$$
\alpha(\lambda)=0 \text { and } L(\lambda)=\operatorname{diag}(U, U, \ldots, U) .
$$

where

$$
U=\left(\begin{array}{ll}
0 & 1 \\
1 & 0
\end{array}\right)
$$

This result is essentially due to Milnor. Using [9, Lemma 9], [17, Theorem 1], and the fact that the linking matrix of a presentation is the same as a matrix of intersection numbers of homology classes in $H_{n}\left(N^{2 n}, Z\right)$ $\left(\approx H_{n}\left(M^{2 n}, Z\right)\right)$, we can find a presentation $\lambda$ of $N^{2 n}$ such that

$$
L(\lambda)=\operatorname{diag}(U, U, \cdots, U), \quad \text { where } U=\left(\begin{array}{ll}
0 & 1 \\
1 & 0
\end{array}\right) .
$$

The fact that $\alpha(\lambda)=0$ then follows immediately from [9, Lemma 7].

Before stating the corresponding result for $n$ odd, some remarks are in order. It is well known (see Albert [1, p. 52]) that every skew symmetric bilinear form of determinant \pm 1 has a matrix representation of the form $\operatorname{diag}(U, U, \cdots, U)$, where

$$
U=\left(\begin{array}{rr}
0 & 1 \\
-1 & 0
\end{array}\right)
$$

Since the linking matrix of a presentation of an element of $\mathscr{H}(2 n, k, n)$ is skew symmetric for $n$ odd and since this matrix will (by Poincaré Duality) have determinant \pm 1 if the handlebody arises by taking the interior of a disk out of a closed $(n-i)$-connected $2 n$-dimensional manifold we know by Wall [17, Theorem 1]: If $M^{2 n}$ is a closed $(n-1)$-connected $2 n$-dimensional differentiable manifold, $n$ odd, and if $D_{0}^{2 n}$ is an embedded disk then $N^{2 n}=M^{2 n}-\operatorname{int} D_{0}^{2 n}$ has a presentation $\lambda$ such that

$$
L(\lambda)=\operatorname{diag}(U, \cdots, U), \quad \text { where } U=\left(\begin{array}{rr}
0 & 1 \\
-1 & 0
\end{array}\right) .
$$

It remains to find the right condition on $M^{2 n}$ to ensure that $\alpha(\lambda)=0$. Assume $n \equiv 3,5,7(\bmod 8), n \neq 3,7$. In these dimensions it follows from results of Bott and Kervaire (see Wall [17, p. 171]) that $\pi_{n-1}(\operatorname{SO}(n)$ ) $\approx Z_{2}$. Thus if $N^{2 n}=M^{2 n}-\operatorname{int} D_{0}^{2 n}$ and $\lambda$ is a presentation of $N^{2 n}$ then 


$$
\alpha(\lambda) \in Z_{\dot{2}} \oplus Z_{\dot{2}} \oplus \ldots \oplus Z_{2} \quad(k \text { terms })
$$

Assume

$$
L(\lambda)=\operatorname{diag}(U, U, \cdots, U), \quad U=\left(\begin{array}{rr}
0 & 1 \\
-1 & 0
\end{array}\right) .
$$

The Arf invariant $\Phi\left(M^{2 n}\right) \in Z_{2}$ of $M^{2 n}$ is now defined by the formula

$$
\Phi\left(M^{2 n}\right)=\sum_{j=1}^{s} \alpha\left(\lambda_{2 j-1}\right) \alpha\left(\lambda_{2 j}\right), \quad \text { where } k=2 s .
$$

It can be shown (see Wall [17, p. 172, Case 6]) that $\Phi\left(M^{2 n}\right)$ does not depend on $\lambda$, i.e., $\Phi\left(M^{2 n}\right)$ is a topological invariant of $M^{2 n}$. Further, it is known that if $\Phi\left(M^{2 n}\right)=0$, then $N^{2 n}$ has a presentation $\lambda$ such that

$$
L(\lambda)=\operatorname{diag}(U, U, \cdots, U), \text { where } U=\left(\begin{array}{rr}
0 & 1 \\
-1 & 0
\end{array}\right) \text { and } \alpha(\lambda)=0
$$

(again see [17]).

The above discussion can be summarized as follows:

Lemma 4. Let $n \equiv 3,5,7(\bmod 8)$. Let $M^{2 n}$ be a closed $(n-1)$-connected 2n-dimensional differentiable manifold and suppose $\Phi\left(M^{2 n}\right)=0$. Let $D_{0}^{2 n}$ be a differentiably embedded disk and let $N^{2 n}=M^{2 n}-\operatorname{int} D_{0}^{2 n}$. Then $N^{2 n}$ has a presentation $\lambda$ such that

$$
\alpha(\lambda)=0 \text { and } L(\lambda)=\operatorname{diag}(U, U, \cdots, U),
$$

where

$$
U=\left(\begin{array}{rr}
0 & 1 \\
-1 & 0
\end{array}\right)
$$

Note that in the statement of Lemma 4 we have included the cases $n=3,7$ although they were excluded from the preceding discussion. This is because $\pi_{2}(\mathrm{SO}(3))=\pi_{6}(\mathrm{SO}(7))=0$ and so $\alpha(\lambda)$ is always zero in these dimensions.

3. Proofs of the main theorems. The proof of Theorem 1 is now completed as follows. Suppose $M^{2 n}$ is a closed $(n-1)$-connected $2 n$-dimensional $n$-parallelizable manifold, $I\left(M^{2 n}\right)=0, n$ even, $n \geqq 6$, and $N^{2 n}=M^{2 n}$ - int $D_{0}^{2 n}$. Then (combining Lemmas 3 and 1) there exists a differentiable embedding $f: N^{2 n} \rightarrow R_{-}^{2 n+1}$ such that $f\left(\partial N^{2 n}\right)$ is contained in $R^{2 n}$. Note that $f \mid \partial N^{2 n}$ is a differentiable embedding of a $(2 n-1)$-sphere in $2 n$-space and so by the Generalized Schoenflies Theorem (see Smale [12, Theorem M]) the closure of the bounded component of $R^{2 n}-f\left(\partial N^{2 n}\right)$ is diffeomorphic to a disk $\bar{D}_{0}^{2 n}$. Lift the interior of this disk off $R^{2 n}$ into $R_{+}^{2 n+1}$ and smooth along $f\left(\partial N^{2 n}\right)$. The result is a differentiable embedding of a closed 
manifold $T^{2 n}$ in $R^{2 n+1}$. In general, $T^{2 n}$ is not diffeomorphic to the manifold $M^{2 n}$ that we started with. $T^{2 n}$ is the union of $N^{2 n}$ with a disk $D_{0}^{2 n}$ attached along $\partial N^{2 n}=S^{2 n-1}$ by a diffeomorphism of $S^{2 n-1}$ onto itself. That is, $T^{2 n}$ is diffeomorphic to the connected sum of $M^{2 n}$ with an element $H^{2 n} \in \theta^{2 n}$ (see Wall [17, bottom of p. 169]). Thus $M^{2 n}$ is almost diffeomorphic to $T^{2 n}$ and so $M^{2 n}$ is almost differentiably embeddable in $R^{2 n+1}$ proving half of Theorem 1 .

To go in the other direction, suppose $M^{2 n}$ is almost differentiably embeddable in $R^{2 n+1}$. Then there exists a differentiable manifold $T^{2 n}$, an almost diffeomorphism $h: M^{2 n} \rightarrow T^{2 n}$, and a differentiable embedding $f$ : $T^{2 n} \rightarrow R^{2 n+1}$. By the Jordan Curve Theorem, $R^{2 n+1}-f\left(T^{2 n}\right)$ has two components. Let $\omega^{2 n+1}$ denote the closure of the bounded component. Then $\omega^{2 n+1}$ is a differentiable manifold with boundary $T^{2 n}$. Therefore, $I\left(T^{2 n}\right)$ $=0$ (see Thom [16, Corollary V. 11] or Milnor [6, Theorem 39]) and so $I\left(M^{i n}\right)=0$ since the index is a topological invariant.

Further, since $\omega^{2 n+1}$ is a $(2 n+1)$-dimensional manifold differentiably embedded in a $(2 n+1)$-space, the tangent bundle $\tau^{2 n+1}$ of $\omega^{2 n+1}$ (being just the restriction of the tangent bundle of $R^{2 n+1}$ to $\omega^{2 n+1}$ ) is trivial. Since $\partial \omega^{2 n+1}=T^{2 n}$, we know that the restriction of $\tau^{2 n+1}$ to $T^{2 n}$ is equal to the Whitney sum of $\tau^{2 n}\left(T^{2 n}\right)$ (the tangent bundle of $T^{2 n}$ ) and a trivial line bundle $\theta^{\prime}$ (the normal bundle of $T^{2 n}$ in $\omega^{2 n+1}$ ). Therefore, $T^{2 n}$ is a $\pi$-manifold. Therefore, $T^{2 n}$ is almost parallelizable (see Milnor [7, Lemma 1.2]). Since $h \mid M^{2 n}-\left\{x_{0}\right\}$ is a diffeomorphism for some point $x_{0} \in M^{2 n}$ we know that $M^{2 n}-\left\{x_{0}\right\}$ is almost parallelizable. Therefore, $M^{2 n}$ is almost parallelizable and so $M^{2 n}$ is $n$-parallelizable, completing the proof of Theorem 1 .

Proof of Corollary 1. Suppose $M^{2 n}$ is a closed $(n-1)$-connected $2 n$ dimensional $\pi$-manifold. Then $M^{2 n}$ is almost parallelizable and so $n$ parallelizable. Since all the Pontrjagin classes of the tangent bundle of a $\pi$-manifold vanish, we known by the Hirzebruch Index Theorem (Milnor [6, Theorem 39]) that $I\left(M^{2 n}\right)=0$. Therefore, by Theorem $1, M^{2 n}$ is almost differentiably embeddable in $R^{2 n+1}$.

To prove the converse, suppose $M^{2 n}$ is almost differentiably embeddable in $R^{2 n+1}$. Then $M^{2 n}$ is almost diffeomorphic to a $\pi$-manifold $T^{2 n}$ (the manifold which is differentiably embeddable in $R^{2 n+1}$ ). Thus $M^{2 n}=T^{2} \# H^{2 n}$ for some $H^{2 n} \in \theta^{2 n}$. But the elements of $\theta^{2 n}$ are all $\pi$-manifolds (see Kervaire and Milnor [5, Theorem 3.1]). The desired result now follows from the fact that the connected sum of two $\pi$-manifolds is again a $\pi$-manifold.

Proof of Theorem 2. Let $M^{2 n}$ be a closed $(n-1)$-connected $2 n$-dimensional differentiable manifold with $\Phi\left(M^{2 n}\right)=0$. The fact that $M^{2 n}$ is almost differentiably embeddable in $R^{2 n+1}(n \equiv 3,5,7,(\bmod 8), n \geqq 5)$ now follows from Lemmas 1 and 4.

To prove the converse, suppose $M^{2 n}$ is almost differentiably embedd- 
able in $R^{2 n+1}$. Then $M^{2 n}$ is almost diffeomorphic to a differentiable manifold $T^{2 n}$ which is differentiably embeddable in $R^{2 n+1}$. It will be shown that $\Phi\left(T^{2 n}\right)=0$ from which it follows that $\Phi\left(M^{2 n}\right)=0$ since the Arf invariant is a topological invariant.

Note that $T^{2 n}$ bounds a parallelizable manifold $\omega^{2 n+1}$ (the closure of the bounded component of the complement of $T^{2 n}$ in $R^{2 n+1}$ ). Therefore, by Milnor [9, Corollary to Theorem 2] $T^{2 n}$ bounds an $(n-1)$-connected differentiable manifold. (In fact, using the Alexander Duality Theorem, it can be shown that $\omega^{2 n+1}$ is itself $(n-1)$-connected.)

The following result due to Kervaire and Milnor (unpublished-see [5] for closely related material) completes the proof of Theorem 2.

Lemma 5. Let $T^{2 n}$ be a closed $(n-1)$-connected $2 n$-dimensional differentiable manifold ( $n$ odd, $n \geqq 3$ ). If $T^{2 n}$ bounds an $(n-1)$-connected $(2 n+1)$-dimensional differentiable manifold, then $T^{2 n}$ has Arf invariant zero.

A sketch of the proof of Lemma 5 in the particular case in which we are interested proceeds as follows: Let $D^{2 n+1}$ be a small disk in the interior of $\omega^{2 n+1}$. Then $N^{2 n+1}=\omega^{2 n+1}-\operatorname{int} D^{2 n+1}$ is a parallelizable manifold with boundary $T^{2 n}-S^{2 n}$. Let $f: N^{2 n+1} \rightarrow R$ be a "nice function" (see Smale [12]). Embed $N^{2 n+1}$ in a high-dimensional Euclidean space such that the last coordinate is given by the nice function $f$. Since $N^{2 n+1}$ is parallelizable, we have normal frames over $N^{2 n+1}$. The nice function can then be looked at as a sequence of framed spherical modifications on $n$-spheres passing from $T^{2 n}$ to $S^{2 n}$. It follows that $\Phi\left(T^{2 n}\right)=0$ since the Arf invariant is invariant under such modifications (see [5]).

Note that as a corollary to Theorem 2 and Lemma 5 we have the following.

Corollary 2. A closed $(n-1)$-connected 2n-dimensional differentiable manifold $M^{2 n}(n \equiv 3,5,7(\bmod 8), n \geqq 5)$ is almost differentiably embeddable in $R^{2 n+1}$ if and only if $M^{2 n}$ is almost diffeomorphic to the boundary of a parallelizable manifold.

This result also holds for $n$ even, $n \geqq 6$, by Theorem 1 .

The problem of extending Corollary 1 to include the cases $n \equiv 3,5,7$ $(\bmod 8), n \geqq 5$, now reduces to answering the following question in the affirmative: Is every $(n-1)$-connected $2 n$-dimensional $\pi$-manifold almost diffeomorphic to the boundary of a parallelizable manifold? To put it another way, what is called for is the converse to the following well-known consequence of Theorem 2 .

Corollary 3. If $n \equiv 3,5,7(\bmod 8), n \geqq 5$, and $M^{2 n}$ is a closed $(n-1)$ connected 2n-dimensional differentiable manifold of Arf invariant zero then $M^{2 n}$ is a $\pi$-manifold. 
Proof. The proof proceeds exactly as does half of the proof of Corollary 1 ; that is, $M^{2 n} \# H^{2 n}$ is a $\pi$-manifold for some $H^{2 n} \in \theta^{2 n}$ and so $M^{2 n}$ is itself a $\pi$-manifold.

4. Some more consequences and final remarks. The following facts are almost immediate consequences of Corollary 1 and the fact that almost diffeomorphic manifolds differ by homotopy spheres.

Corollary 4. Let $n$ be even, $n \geqq 6$. Suppose $\theta^{2 n}=0$ (for example, $n=6$ ). Then a closed $(n-1)$-connected $2 n$-dimensional differentiable manifold is differentiably embeddable in $R^{2 n+1}$ if and only if it is a $\pi$-manifold.

Proof. $\theta^{2 n}=0$ means that the $2 n$-sphere has a unique differentiable structure. In this case, every almost diffeomorphism is a diffeomorphism and every almost differentiable embedding a differentiable embedding.

CoROLlary 5 . Let $n$ be even, $n \geqq 6$. Let $R^{m(n)}$ be the smallest-dimensional Euclidean space in which every homotopy $2 n$-sphere is differentiably embeddable. Then every closed $(n-1)$-connected $2 n$-dimensional $\pi$-manifold is differentiably embeddable in $R^{m(n)}$.

Proof. If $M^{2 n}$ is a closed $(n-1)$-connected $\pi$-manifold, then there exists $H^{2 n} \in \theta^{2 n}$ such that $M^{2 n} \# H^{2 n}$ is differentiably embeddable in $R^{2 n+1} \subset R^{m(n)}$. Now $\left(M^{2 n} \# H^{2 n}\right) \#\left(-H^{2 n}\right)$ is diffeomorphic to $M^{2 n}$, where $-H^{2 n}$ is the group inverse of $H^{2 n}$. The desired result now follows from the fact that if each of two manifolds is differentiably embeddable in a given Euclidean space, then so is their connected sum.

Analogous results hold for $n \equiv 3,5,7(\bmod 8), n \geqq 5$.

The following is worth noting here.

Proposition. If $M^{2 n}$ is a closed $(n-1)$-connected $2 n$-dimensional differentiable manifold and $n \equiv 3,5,6,7(\bmod 8)$, then $M^{2 n}$ is $n$-parallelizable.

Proof. Let $\tau: M^{2 n} \rightarrow \mathrm{BSO}(2 n)$ be the map which defines the tangent bundle of $M^{2 n}$. Let $K^{n} \subset M^{2 n}$ be the $n$ skeleton. Then $M^{2 n}$ is $n$-parallelizable if and only if $\tau \mid K^{n}$ is homotopic to the constant map. Since $M^{2 n}$ is $(n-1)$-connected, $K^{n}$ is a wedge of spheres. Therefore $\tau \mid K^{n}$ can be looked at as an element of $\pi_{n}(\mathrm{BSO}(2 n)) \approx \pi_{n-1}(\mathrm{SO}(2 n))=0$ (see Bott [2]) and so $M^{2 n}$ is $n$-parallelizable.

Thus $n$-parallelizability is implicit in Theorem 2 and redundant in the statement of Theorem 1 for $n \equiv 6(\bmod 8)$.

The techniques described in this paper can be used to give a new proof of a result due to Hirsch (see [4, Corollary 4.3]).

Theorem 3. Let $M^{2 n}$ be a closed $(n-1)$-connected $2 n$-dimensional differentiable manifold $n \equiv 3,5,7,(\bmod 8), n \geqq 5$. Let $x_{0} \in M^{2 n}$. Then $M^{2 n}-\left\{x_{0}\right\}$ can be differentiably embedded in $R^{2 n+1}$. 
Proof. As always, let $D_{0}^{2 n} \subset M^{2 n}$ be a small disk with center $x_{0}$ and $N^{2 n}$ $=M^{2 n}-\operatorname{int} D_{0}^{2 n}$. It will be shown that $N^{2 n}$ (and therefore $\left.M^{2 n}-\left\{x_{0}\right\}\right)$ is differentiably embeddable in $R^{2 n+1}$.

Let $\lambda$ be a presentation of $N^{2 n}$ such that

$$
L(\lambda)=\operatorname{diag}(U, U, \cdots, U), \quad U=\left(\begin{array}{rr}
0 & 1 \\
-1 & 0
\end{array}\right), \quad N^{2 n}=D^{2 n} \cup_{\lambda} D_{k} .
$$

As in Lemma 1, we can find an embedding

$$
f^{\prime}: D^{2 n} \cup_{\lambda} \bigcup_{i=1}^{k} D_{i}^{n} \times 0 \rightarrow R_{-}^{2 n+1}
$$

such that $f^{\prime}\left(\partial D^{2 n} \cup_{\lambda} \bigcup_{i=1}^{k} D_{i}^{n} \times 0\right) \subset R^{2 n}$.

Now, however, we can no longer thicken the cores $\left\{f^{\prime}\left(D_{i}^{n} \times 0\right)\right\}$ of the handles in $R^{2 n}$ since we have removed the restriction $\alpha(\lambda)=0$. We can, however, embed $D_{k}$ in $R^{2 n+1}$ so that the embedding extends $f^{\prime} \mid C_{k} \cup \bigcup_{i=1}^{k} D_{i}^{n}$ $\times 0$. This is done as follows: Observe that the obstruction to thickening $f^{\prime}\left(D_{i}^{n} \times 0\right)$ in $R^{2 n+1}$ (i.e., the obstruction to extending the field of $n$ frames in the normal tube over $f^{\prime}\left(\lambda\left(\partial D_{i}^{n} \times 0\right)\right)$ given by $f^{\prime}\left(\lambda\left(\partial D_{i}^{n} \times D^{n}\right)\right)$ to a field of $n$ frames in the normal tube of $f^{\prime}\left(D_{i}^{n} \times 0\right)$ in $\left.R^{2 n+1}\right)$ is an element of $\pi_{n-1}\left(V_{n+1, n}\right) \approx \pi_{n-1}(\operatorname{SO}(n+1)) \approx \pi_{n-1}(\operatorname{SO}(2 n))$ which is the zero group if $n \equiv 3,5,6,7(\bmod 8)$. Thus $N^{2 n}$ is embeddable in $R^{2 n+1}$.

ADDENDA. (1) Since this writing, I have been informed that by using results of $\mathrm{Wu}$ rather than those of Haefliger, the bound in Lemma 2 can be lowered to $n \geqq 3$. Therefore, the bound in Theorem 1 can be lowered to $n \geqq 4$ and in Theorem 2 to $n \geqq 3$.

(2) If $n \equiv 1(\bmod 8)$ we have that $\pi_{n-1}(\operatorname{SO}(n))$ is isomorphic to $Z_{2} \oplus Z_{2}$. The extra $Z_{2}$ necessitates the introduction of second invariant $X$ (see Wall [ 17, bottom of p. 172]). Results similar to Theorem 2 can be obtained in this case involving $\Phi$ and $X$.

I am grateful to Professors M. W. Hirsch and R. K. Lashof for their generous help and stimulation during this work. This research was partially financed by National Science Foundation Grant GP-812.

\section{REFERENCES}

1. A. A. Albert, Introduction to algebraic theories, Univ. of Chicago Press, Chicago, Ill., 1941.

2. R. Bott, The stable homotopy of the classical groups, Proc. Nat. Acad. Sci. U. S. A. 43 (1957), 933-935.

3. A. Haefliger, Plongements différentiables de variétés dans variétés, Comment. Math. Helv. 36 (1961), 47-81.

4. M. W. Hirsch, On embedding differentiable manifolds in Euclidean space, Ann. of Math. (2) 73 (1961), 566-571.

5. M. Kervaire and J. Milnor, Groups of homotopy spheres. I, mimeographed, New York University, Courant Institute of Mathematical Sciences, December, 1961.

6. J. Milnor, Lectures on characteristic classes, mimeographed, Princeton University, Princeton, 
N. J., 1957.

7. Differentiable manifolds which are homotopy spheres, mimeographed, Princeton University, Princeton, N. J., 1959.

8. —, Morse theory. I, mimeographed, Princeton University, Princeton, N. J., 1960.

9. __ A procedure for killing homotopy groups of differentiable manifolds, Proc. Sympos. Pure Math. Vol. 3, pp. 39-55, Amer. Math. Soc., Providence, R. I., 1961.

10. J. Munkres, Obstructions to the smoothing of piecewise differentiable homeomorphisms, Ann. of Math. (2) 72 (1960), 521-554.

11. H. Seifert and W. Threlfall, Lehrbuch der Topologie, Chelsea, New York, 1947.

12. S. Smale, Generalized Poincare's conjecture in dimensions greater than four, Ann. of Math. (2) 74 (1961), 391-406.

13. __ Differentiable and combinatorial structures on manifolds, Ann. of Math. (2) 74 (1961), 498-502.

14. _ On the structure of 5 manifolds, Ann. of Math. (2) 75 (1962), 38-46.

15. N. Steenrod, Topology of fiber bundles, Princeton Univ. Press, Princeton, N. J., 1951.

16. R. Thom, Espaces fibres en sphères et carrés de Steenrod, Ann. Sci. École Norm. Sup. 69 (1952), 109-181.

17. C. T. C. Wall, Classification of (n-1)-connected 2n-manifolds, Ann. of Math. (2) 75 (1962), 163-189.

\author{
University OF Chicago, \\ Chicago, ILuinois \\ UNIVERSITY OF CALIFORNIA, \\ Berkeley, CALIFORNIA
}

